\title{
DECOUPLED ENERGY STABLE SCHEMES FOR PHASE-FIELD VESICLE MEMBRANE MODEL
}

\author{
$\mathrm{RUI} \mathrm{CHEN}^{\dagger}, \mathrm{GUANGHUA} \mathrm{JI}^{\dagger}$, XIAOFENG YANG ${ }^{\ddagger}$ AND HUI ZHANG ${ }^{\dagger}$
}

\begin{abstract}
We consider the numerical approximations of the classical phase-field vesicle membrane models proposed a decade ago in [450, 198 (2004), J. Comput. Phys.]. We first reformulate the model derived from an energetic variational formulation into a form which is suitable for numerical approximation, and establish the energy dissipation law. Then, we develop a stabilized, decoupled, time discretization scheme for the coupled nonlinear system. The scheme is unconditionally energy stable and leads to linear and decoupled elliptic equations to be solved at each time step. Stability analysis and ample numerical simulations are presented thereafter.
\end{abstract}

\section{INTRODUCTION}

In cell biology, a vesicle is a small organelle within a cell, consisting of fluid enclosed by a lipid bilayer membrane. There have been many experimental and analytic studies on the configurations and deformations of elastic vesicle bio-membranes $[2,6,12,14,15,18]$. In the last decade, using the energetic, variational diffuse interface approach, Du. et al. proposed a phase-field model to simulate the deformations of simple vesicles coupled with incompressible flow fields $[6-8,10]$, in which, the Helfrich bending elastic energy of the surface is replaced by a phase field functional. The evolution equations are then resulted from the variations of the action functional of the free energy.

The diffuse-interface/phase-field models, whose origin can be traced back to [9,34], have been proved efficient with much success. A particular advantage of the phase-field approach is that they can often be derived from an energy-based variational formalism, leading to well-posed nonlinear coupled systems that satisfy thermodynamics-consistent energy dissipation laws. Thus it is especially desirable to design numerical schemes that preserve the energy dissipation law at the discrete level. Due to the rapid changes near the interface, the non-compliance of energy dissipation laws of the numerical scheme may lead to spurious numerical solutions if the grid and time step sizes are not carefully controlled $[11,22]$. Another main advantage of energy stable schemes is that they can be easily combined with an adaptive time stepping strategy [23-25, 28, 37].

To construct the numerical schemes for the typical phase-field models coupled with the hydrodynamics, in particular, the Allen-Cahn or Cahn-Hilliard equations, the main difficulties include (i) the coupling between the velocity and phase function through the convection term in the phase equation and nonlinear stress in the momentum equation; (ii) the coupling of the velocity and pressure through the incompressibility constraint; (iii) the stiffness of the phase equation associated with the interfacial width. For the phase-field vesicle membrane model $[6-8,10]$, things are about to get even worse due to some extra nonlinear terms with second order derivatives. To the best of

${ }^{1 \dagger}$ School of Mathematical Sciences, Beijing Normal University, Laboratory of Mathematics and Complex Systems, Ministry of Education, Beijing 100875, P.R. China, (ruichenbnu@gmail.com, ghji@bnu.edu.cn, hzhang@bnu.edu.cn). $¥$ Department of Mathematics, University of South Carolina, Columbia, SC, 29208, USA. (xfyang@math.sc.edu). Key words and phrases. Phase-field, Multiphase flows, Vesicle Membrane, Navier-Stokes, Allen-Cahn, Stability. 
the authors' knowledge, there does not exist any easy-to-implement and energy stable scheme for this model so far.

Thus, for the phase-field membrane vesicle model, the main purpose of this paper is to construct a time discretization scheme which (a) satisfies a discrete energy law; and (b) leads to decoupled elliptic equations to solve at each time step. This is by no means an easy task due to many highly nonlinear terms and the couplings among the velocity, pressure and phase function.

The rest of the paper is organized as follows. In section 2, we introduce the phase-field vesicle membrane model and derive the energy dissipation law. In section 3, we reformulate the PDE to an equivalent form, construct a decoupled, energy stable numerical scheme, and give the stability analysis. In section 4 , we present the spatial discretization using the finite element method. In section 5 , we present some numerical results to illustrate the accuracy and efficiency of the proposed scheme and summarize our contributions. Some concluding remarks are given in section 6 .

\section{MODELS}

The equilibrium shape of a vesicle membrane is determined by minimizing the elastic bending energy $[3,4]$,

$$
E=\int_{\Gamma}\left(a_{1}+a_{2}\left(H-c_{0}\right)^{2}+a_{3} K\right) d s
$$

where $H=\frac{k_{1}+k_{2}}{2}$ represents mean curvature of the membrane surface; $K=k_{1} k_{2}$ is Gaussian curvature; $k_{1}, k_{2}$ are two principle curvatures; $a_{1}$ is the surface tension; $a_{2}, a_{3}$ are bending rigidities; $c_{0}$ represents spontaneous curvature; $\Gamma$ is a smooth compact surface in the domain $\Omega \in \mathbf{R}^{3}$.

If we consider the model to be isotropic, i.e., the spontaneous curvature $c_{0}=0$ and neglect the constants $a_{1}$ and $a_{3}$ due to the Gauss-Bonnet formula, then the elastic bending energy can be written by,

$$
E=\int_{\Gamma} \frac{K}{2} H^{2} d s
$$

In the framework of phase-field method, a variable $\phi(x)=\tanh \left(\frac{d(x)}{\sqrt{2} \epsilon}\right)$ is defined for all $x \in \Omega$, where $d(x)$ is the signed distance between a point $x$ and $\Gamma$, positive inside and negative outside; $\epsilon$ is a transition parameter that is taken to be very small. Thus $H=-\frac{1}{2} \operatorname{tr}\left(\nabla^{2} d(x)\right)$ on the surface and one can obtain the bending energy as follows [8],

$$
E_{b}=\int_{\Omega} \frac{\epsilon}{2}|\Delta \phi-f(\phi)|^{2} d x
$$

where $F(\phi)=\frac{\left(\phi^{2}-1\right)^{2}}{4 \epsilon^{2}}$ is the Ginzburg-Landau double well potential, $f(\phi)=F^{\prime}(\phi)$ and $\epsilon$ is penalty parameter.

If one considers the constrains of the volume and the surface area, then the energy functional $E_{b}$ includes two extra terms as follows $[6,10]$,

$$
E_{b}=\int_{\Omega} \frac{\epsilon}{2}|\Delta \phi-f(\phi)|^{2} d x+\frac{1}{2} M_{1}(A(\phi)-\alpha)^{2}+\frac{1}{2} M_{2}(B(\phi)-\beta)^{2},
$$


where

$$
A(\phi)=\int_{\Omega} \phi(x) d x, \quad B(\phi)=\int_{\Omega} \epsilon\left(\frac{1}{2}|\nabla \phi|^{2}+F(\phi)\right) d x,
$$

where $A(\phi)$ denotes the volume fractions, $B(\phi)$ is approaching a value of $2 \sqrt{2} / 3$ times of superficial area of the phase, $M_{1}$ and $M_{2}$ are the positive penalty parameters, $\alpha$ and $\beta$ denote the constants of the volume and surface area, respectively.

Assuming the system is a vesicle bounded by incompressible fluid flows, the total energy $E_{t o t}$ of the hydrodynamic system is a sum of the kinetic energy $E_{k}$ and the bending energy $E_{b}$, i.e.,

$$
E_{\text {tot }}=E_{k}+\lambda E_{b}=\int_{\Omega} \frac{1}{2} \rho|\mathbf{u}|^{2} d x+\lambda E_{b},
$$

where $\rho$ is the density, $\mathbf{u}$ is the velocity, $\lambda$ is the surface tension parameter.

Assuming a generalized Fick's law that the mass flux is proportional to the gradient of the chemical potential, one can derive the following system of Allen-Cahn type,

$$
\begin{aligned}
& \phi_{t}+\mathbf{u} \cdot \nabla \phi=-M \lambda \frac{\delta E_{b}}{\delta \phi}, \\
& \rho\left(\mathbf{u}_{t}+\mathbf{u} \cdot \nabla \mathbf{u}\right)-\nu \Delta \mathbf{u}+\nabla p-\lambda \frac{\delta E_{b}}{\delta \phi} \nabla \phi=0, \\
& \nabla \cdot \mathbf{u}=0,
\end{aligned}
$$

where $p$ is the pressure, $\nu$ is the viscosity, $M$ is the relaxation time scale.

The variational derivative is

$$
\begin{aligned}
\frac{\delta E_{t o t}}{\delta \phi}= & \lambda \epsilon\left(\Delta^{2} \phi-\Delta f(\phi)-f^{\prime}(\phi) \Delta \phi+f^{\prime}(\phi) f(\phi)\right) \\
& +\lambda M_{1}(A(\phi)-\alpha)-\lambda \epsilon M_{2}(B(\phi)-\beta)(\Delta \phi-f(\phi)) .
\end{aligned}
$$

Throughout the paper, we assume the following boundary conditions

$$
\left.\mathbf{u}\right|_{\partial \Omega}=0,\left.\quad \frac{\partial \phi}{\partial \mathbf{n}}\right|_{\partial \Omega}=0,\left.\quad \frac{\partial \Delta \phi}{\partial \mathbf{n}}\right|_{\partial \Omega}=0,
$$

where $\mathbf{n}$ denotes the outward normal of the boundary.

By taking the inner product of (2.7) with $\frac{\delta E_{t o t}}{\delta \phi},(2.8)$ with $\mathbf{u}$, and then summing up these equalities, we obtain the energy dissipation law as follows,

$$
\partial_{t} E_{t o t}=-\int_{\Omega}\left(\nu|\nabla \mathbf{u}|^{2}+M\left|\frac{\delta E_{t o t}}{\delta \phi}\right|^{2}\right) d x .
$$

The above energy laws enable one to prove the existence and uniqueness of the weak solution with certain smoothness by a standard Galerkin procedure [3].

\section{Alternative formulation and its Decoupled energy stable scheme}

The coupled nonlinear system (2.7)-(2.9) actually presents formidable challenges for algorithm design, implementation as well as numerical analysis. Although many numerical schemes perform well in practice, the question of their stability remains open [8]. The emphasis of our algorithm development is placed on designing numerical schemes that are not only easy-to-implement, but also satisfy a discrete energy dissipation law. We will design schemes that in particular can overcome the following difficulties, including, the coupling of the velocity and pressure through the incompressible 
condition; the stiffness in the phase equation associated with the interfacial width $\epsilon$; and the nonlinear couplings between the fluid equation and the phase equation through the convection terms as well as the stresses. To the best of the authors' knowledge, this is the first such scheme for the phase field vesicle membrane model.

3.1. Alternative formulations and energy law. While it is straightforward to derive the energy law (2.12) by taking the inner product of (2.7) with $\frac{\delta E_{b}}{\delta \phi}$ and (2.8) with $\mathbf{u}$. However, the nonlinear term in $\frac{\delta E_{b}}{\delta \phi}$ involves fourth order and second order derivatives, and it is not convenient to use them as test functions in numerical approximations, making it difficult to prove energy dissipation law in the discrete level.

Thus, to overcome this difficulty, we have to reformulate the system (2.7)-(2.9) in an alternative form which is convenient for numerical approximation. Throughout the paper, we assume $\rho=1$ for simplicity. We let $\dot{\phi}=\phi_{t}+\mathbf{u} \cdot \nabla \phi$, then the system (2.7) -(2.9) can be rewritten as follows.

$$
\begin{aligned}
& \frac{1}{M} \dot{\phi}=-\lambda \frac{\delta E_{b}}{\delta \phi}, \\
& \mathbf{u}_{t}+\mathbf{u} \cdot \nabla \mathbf{u}-\nu \Delta \mathbf{u}+\nabla p+\frac{1}{M} \dot{\phi} \nabla \phi=0 \\
& \nabla \cdot \mathbf{u}=0 .
\end{aligned}
$$

We now show that the above system admits an energy law. Taking the inner products of (3.1) with $\phi_{t}$, and of (3.2) with $\mathbf{u}$, we derive

$$
\frac{1}{M}\|\dot{\phi}\|^{2}-\frac{1}{M}(\dot{\phi}, \mathbf{u} \cdot \nabla \phi)=-\lambda \partial_{t} E_{b}
$$

and

$$
\frac{1}{2} \partial_{t}\|\mathbf{u}\|^{2}+\nu\|\nabla \mathbf{u}\|^{2}+\frac{1}{M}(\dot{\phi} \nabla \phi, \mathbf{u})=0 .
$$

Summing up the above equalities, we have the same energy law as (2.12).

$$
\partial_{t} E_{t o t}=\int_{\Omega}\left(-\nu|\nabla \mathbf{u}|^{2}-\frac{1}{M}|\dot{\phi}|^{2}\right) d \mathbf{x}
$$

We emphasize that the above derivation is suitable in a finite dimensional approximation since the test function $\phi_{t}$ is in the same subspaces as $\phi$. Hence, it allows us to design numerical schemes which satisfy the energy dissipation law in the discrete level.

3.2. Energy stable scheme. We construct an energy stable scheme based on the stabilization approach $[30,35]$. To this end, we shall assume the functional $F(x)$ satisfies the following conditions: there exist constants $L_{1}$ and $L_{2}$ depending on $\epsilon$ such that

$$
\max _{x \in \mathbb{R}}\left|f^{\prime}(x)\right| \leq L_{1}, \quad \max _{x \in \mathbb{R}}\left|f^{\prime \prime}(x)\right| \leq L_{2} .
$$

One immediately notes that this condition is not satisfied by the usual Ginzburg-Landau double well potential $F(\phi)=\frac{1}{4 \epsilon^{2}}\left(\phi^{2}-1\right)^{2}$. However, we can truncate $F(\phi)$ to quadratic growth outside of an interval $[-N, N]$ without affecting the solution if the maximum norm of the initial condition $\phi_{0}$ is bounded by $N$. 
Here we specially remark that the assumption (3.7) is important for constructing the energy stable scheme. In fact, the function $\phi$ is bounded from viewpoint of physics, so the assumption (3.7) is physically reasonable, although the most commonly used double well potential does not satisfy mathematically.

For simplicity, we consider the bending energy in (2.3) without the surface area constraint, i.e. the system (2.10) with $M_{2}=0$.

Our numerical scheme reads as follows.

Given the initial conditions $\phi^{0}, \mathbf{u}^{0}$ and $p^{0}=0$, having computed for $\phi^{n}, \mathbf{u}^{n}, p^{n}$ for $n>0$, we compute $\phi^{n+1}, \mathbf{u}^{n+1}, p^{n+1}$ by

Step 1:

$$
\begin{aligned}
& \frac{1}{M} \dot{\phi}^{n+1}+C_{1}^{n}\left(\phi^{n+1}-\phi^{n}\right)-C_{2}^{n} \Delta\left(\phi^{n+1}-\phi^{n}\right) \\
& \quad+\lambda \epsilon\left(\Delta^{2} \phi^{n+1}-\Delta f\left(\phi^{n}\right)-f^{\prime}\left(\phi^{n}\right) \Delta \phi^{n}+f^{\prime}\left(\phi^{n}\right) f\left(\phi^{n}\right)\right)+\lambda M_{1}\left(A\left(\phi^{n}\right)-\alpha\right)=0,
\end{aligned}
$$

with

$$
\begin{aligned}
& \dot{\phi}^{n+1}=\frac{\phi^{n+1}-\phi^{n}}{\delta t}+\mathbf{u}_{\star}^{n} \cdot \nabla \phi^{n}, \\
& \mathbf{u}_{\star}^{n}=\mathbf{u}^{n}-\delta t \frac{1}{M} \dot{\phi}^{n+1} \nabla \phi^{n} .
\end{aligned}
$$

Step 2:

$$
\begin{aligned}
& \frac{\tilde{\mathbf{u}}^{n+1}-\mathbf{u}_{\star}^{n}}{\delta t}+\left(\mathbf{u}^{n} \cdot \nabla\right) \tilde{\mathbf{u}}^{n+1}-\nu \Delta \tilde{\mathbf{u}}^{n+1}+\nabla p^{n}=0, \\
& \left.\tilde{\mathbf{u}}^{n+1}\right|_{\partial \Omega}=0 .
\end{aligned}
$$

Step 3:

$$
\begin{aligned}
& \frac{\mathbf{u}^{n+1}-\tilde{\mathbf{u}}^{n+1}}{\delta t}+\nabla\left(p^{n+1}-p^{n}\right)=0, \\
& \nabla \cdot \mathbf{u}^{n+1}=0, \\
& \left.\mathbf{u}^{n+1} \cdot \mathbf{n}\right|_{\partial \Omega}=0 .
\end{aligned}
$$

In the above, $C_{1}^{n}$ and $C_{2}^{n}$ are two stabilizing parameters to be determined.

Several remarks are in order:

- A pressure-correction scheme is used to decouple the computation of the pressure from that of the velocity.

- We recall that $f(\phi)=\frac{1}{\epsilon^{2}} \phi\left(\phi^{2}-1\right)$, so the explicit treatment of this term usually leads to a severe restriction on the time step $\delta t$ when $\epsilon \ll 1$. Thus we introduce in (3.8) two "stabilizing" terms to improve the stability while preserving the simplicity. It allows us to treat all nonlinear terms explicitly without suffering from any time step constraint $[16,17$, 31 . Note that this stabilizing term introduces an extra consistent error of order $O(\delta t)$ in a small region near the interface, but this error is of the same order as the error introduced by treating $f(\phi)$ explicitly, so the overall truncation error is essentially of the same order with or without the stabilizing term. 
- We introduce new, explicit, convective velocities $\mathbf{u}_{\star}^{n}$ in the phase equation. It can be computed directly from (3.9), i.e.

$$
\mathbf{u}_{\star}^{n}=\left(I+\frac{\delta t}{M} \nabla \phi^{n} \nabla \phi^{n}\right)^{-1}\left(\mathbf{u}^{n}-\frac{\phi^{n+1}-\phi^{n}}{M} \nabla \phi^{n}\right),
$$

It is easy to get the $\operatorname{det}(I+c \nabla \phi \nabla \phi)=1+c \nabla \phi \cdot \nabla \phi$, thus the above matrix is invertible. The similar convective term was used in $[1,20,31,32]$ for a phase-field model of three-phase Newtonian fluids and two-phase complex fluids system, respectively.

- The scheme (3.8)-(3.12) is first order in time. It is totally decoupled and linear scheme. (3.12) can be reformulated as a Poisson equation for $p^{n+1}-p^{n}$. (3.8) can be reformulated into two second order elliptic equations that is shown in section 4 . Therefore, at each time step, one only needs to solve a sequence of decoupled elliptic equations which can be solved very efficiently.

- As we shall show below, the above scheme is unconditionally energy stable. To the best of the authors' knowledge, this is the first such scheme for the phase-field fluid vesicle membrane model since it was developed a decade ago.

Theorem 3.1. Under the conditions,

$$
\begin{aligned}
& C_{1}^{n} \geq \frac{1}{2} L_{1}^{2} \lambda \epsilon+\frac{1}{2} L_{2} \lambda \epsilon\left\|\Delta \phi^{n}\right\|_{\infty}+\frac{1}{2} L_{2} \lambda \epsilon\left\|f\left(\phi^{n}\right)\right\|_{\infty}+\frac{1}{2} \lambda M_{1}|\Omega|, \\
& C_{2}^{n} \geq L_{1} \lambda \epsilon,
\end{aligned}
$$

the scheme (3.8)-(3.12) admits a unique solution satisfying the following discrete energy dissipation law

$$
\begin{aligned}
\frac{1}{2}\left\|\mathbf{u}^{n+1}\right\|^{2}+\lambda E_{b}^{n+1}+\frac{1}{2} \delta t^{2}\left\|\nabla p^{n+1}\right\|^{2} & +\left\{\nu \delta t\left\|\nabla \tilde{\mathbf{u}}^{n+1}\right\|^{2}+\delta t \frac{1}{M}\left\|\dot{\phi}^{n+1}\right\|^{2}\right\} \\
& \leq \frac{1}{2}\left\|\mathbf{u}^{n}\right\|^{2}+\lambda E_{b}^{n}+\frac{1}{2} \delta t^{2}\left\|\nabla p^{n}\right\|^{2}
\end{aligned}
$$

where $E_{b}^{n}=\frac{1}{2} \epsilon\left\|\Delta \phi^{n}-f\left(\phi^{n}\right)\right\|^{2}+\frac{M_{1}}{2}\left(A\left(\phi^{n}\right)-\alpha\right)^{2}$.

Proof. Since every step in the scheme (3.8)-(3.12) consists of a linear elliptic equation, it is easy to see that the scheme is uniquely solvable for $\phi \in H^{2}(\Omega), \tilde{\mathbf{u}}^{n+1} \in H_{0}^{1}(\Omega)^{d i m}, p^{n+1} \in H^{1}(\Omega) \backslash \mathbb{R}$, and $\mathbf{u}^{n+1} \in L^{2}(\Omega)^{\operatorname{dim}}$.

By taking the inner product of (3.11) with $\delta t \tilde{\mathbf{u}}^{n+1}$, and using the identity

$$
2(a-b, a)=|a|^{2}-|b|^{2}+|a-b|^{2},
$$

we obtain

$$
\frac{1}{2}\left\|\tilde{\mathbf{u}}^{n+1}\right\|^{2}-\frac{1}{2}\left\|\mathbf{u}_{\star}^{n}\right\|^{2}+\frac{1}{2}\left\|\tilde{\mathbf{u}}^{n+1}-\mathbf{u}_{\star}^{n}\right\|^{2}+\nu \delta t\left\|\nabla \tilde{\mathbf{u}}^{n+1}\right\|^{2}+\delta t\left(\nabla p^{n}, \tilde{\mathbf{u}}^{n+1}\right)=0 .
$$

To deal with the pressure term, we take the inner product of (3.12) with $\delta t^{2} \nabla p^{n}$ to derive

$$
\frac{1}{2} \delta t^{2}\left(\left\|\nabla p^{n+1}\right\|^{2}-\left\|\nabla p^{n}\right\|^{2}-\left\|\nabla p^{n+1}-\nabla p^{n}\right\|^{2}\right)=\delta t\left(\tilde{\mathbf{u}}^{n+1}, \nabla p^{n}\right) .
$$

By taking the inner product of (3.12) with $\frac{1}{2} \mathbf{u}^{n+1}$, we obtain

$$
\frac{1}{2}\left\|\mathbf{u}^{n+1}\right\|^{2}+\frac{1}{2}\left\|\mathbf{u}^{n+1}-\frac{1}{2} \tilde{\mathbf{u}}^{n+1}\right\|^{2}=\frac{1}{2}\left\|\tilde{\mathbf{u}}^{n+1}\right\|^{2} .
$$


We also derive from (3.12) directly that

$$
\frac{1}{2} \delta t^{2}\left\|\nabla p^{n+1}-\nabla p^{n}\right\|^{2}=\frac{1}{2}\left\|\tilde{\mathbf{u}}^{n+1}-\mathbf{u}^{n+1}\right\|^{2} .
$$

Combining all identities above, we obtain

$$
\begin{aligned}
\frac{1}{2}\left\|\mathbf{u}^{n+1}\right\|^{2}-\frac{1}{2}\left\|\mathbf{u}_{\star}^{n}\right\|^{2}+\frac{1}{2}\left\|\tilde{\mathbf{u}}^{n+1}-\mathbf{u}_{\star}^{n}\right\|^{2} & +\frac{1}{2} \delta t^{2}\left(\left\|\nabla p^{n+1}\right\|^{2}-\left\|\nabla p^{n}\right\|^{2}\right) \\
& +\nu \delta t\left\|\nabla \tilde{\mathbf{u}}^{n+1}\right\|^{2}=0 .
\end{aligned}
$$

Next, we derive from (3.10) that

$$
\frac{\mathbf{u}_{\star}^{n}-\mathbf{u}^{n}}{\delta t}=-\frac{1}{M} \dot{\phi}^{n+1} \nabla \phi^{n},
$$

By taking the inner product of $(3.22)$ with $\delta t \mathbf{u}_{\star}^{n}$, we obtain

$$
\frac{1}{2}\left\|\mathbf{u}_{\star}^{n}\right\|^{2}-\frac{1}{2}\left\|\mathbf{u}^{n}\right\|^{2}+\frac{1}{2}\left\|\mathbf{u}_{\star}^{n}-\mathbf{u}^{n}\right\|^{2}=-\frac{1}{M} \delta t\left(\dot{\phi}^{n+1} \nabla \phi^{n}, \mathbf{u}_{\star}^{n}\right),
$$

Combining (3.21) and (3.23), we arrive at

$$
\begin{aligned}
\frac{1}{2}\left\|\mathbf{u}^{n+1}\right\|^{2} & -\frac{1}{2}\left\|\mathbf{u}^{n}\right\|^{2}+\frac{1}{2}\left\|\mathbf{u}_{\star}^{n}-\mathbf{u}^{n}\right\|^{2}+\frac{1}{2}\left\|\tilde{\mathbf{u}}^{n+1}-\mathbf{u}_{\star}^{n}\right\|^{2} \\
& +\frac{1}{2} \delta t^{2}\left(\left\|\nabla p^{n+1}\right\|^{2}-\left\|\nabla p^{n}\right\|^{2}\right)+\nu \delta t\left\|\nabla \tilde{\mathbf{u}}^{n+1}\right\|^{2}=-\frac{1}{M} \delta t\left(\dot{\phi}^{n+1} \nabla \phi^{n}, \mathbf{u}_{\star}^{n}\right) .
\end{aligned}
$$

Then, by taking the inner product of (3.8) with $\left(\phi^{n+1}-\phi^{n}\right)$, we obtain

$$
\begin{aligned}
C_{1}^{n}\left\|\phi^{n+1}-\phi^{n}\right\|^{2} & +C_{2}^{n}\left\|\nabla \phi^{n+1}-\nabla \phi^{n}\right\|^{2}+\delta t \frac{1}{M}\left\|\dot{\phi}^{n+1}\right\|^{2}-\delta t \frac{1}{M}\left(\dot{\phi}^{n+1},\left(\mathbf{u}_{\star}^{n} \cdot \nabla\right) \phi^{n}\right) \\
& +\lambda \epsilon\left(\Delta^{2} \phi^{n+1}, \phi^{n+1}-\phi^{n}\right)(: I) \\
& -\lambda \epsilon\left(\Delta f\left(\phi^{n}\right)+f^{\prime}\left(\phi^{n}\right) \Delta \phi^{n}, \phi^{n+1}-\phi^{n}\right)(: I I) \\
& +\lambda \epsilon\left(f^{\prime}\left(\phi^{n}\right) f\left(\phi^{n}\right), \phi^{n+1}-\phi^{n}\right)(: I I I) \\
& +\lambda M_{1}\left(\left(A\left(\phi^{n}\right)-\alpha\right), \phi^{n+1}-\phi^{n}\right)(: I V) \\
& =0 .
\end{aligned}
$$

For term $I$, we derive

$$
I:=\left(\Delta^{2} \phi^{n+1}, \phi^{n+1}-\phi^{n}\right)=\frac{1}{2}\left(\left\|\Delta \phi^{n+1}\right\|^{2}-\left\|\Delta \phi^{n}\right\|^{2}+\left\|\Delta \phi^{n+1}-\Delta \phi^{n}\right\|^{2}\right) .
$$

For term $I I$, applying the following Taylor expansions

$$
\begin{aligned}
& f\left(\phi^{n+1}\right)-f\left(\phi^{n}\right)=f^{\prime}\left(\phi^{n}\right)\left(\phi^{n+1}-\phi^{n}\right)+\frac{f^{\prime \prime}(\xi)}{2}\left(\phi^{n+1}-\phi^{n}\right)^{2}, \\
& f\left(\phi^{n}\right)-f\left(\phi^{n+1}\right)=-f^{\prime}(\eta)\left(\phi^{n+1}-\phi^{n}\right),
\end{aligned}
$$


we can derive

$$
\begin{aligned}
I I:= & \left(\Delta f\left(\phi^{n}\right)+f^{\prime}\left(\phi^{n}\right) \Delta \phi^{n}, \phi^{n+1}-\phi^{n}\right) \\
= & \left(f\left(\phi^{n}\right), \Delta \phi^{n+1}-\Delta \phi^{n}\right)+\left(f^{\prime}\left(\phi^{n}\right)\left(\phi^{n+1}-\phi^{n}\right), \Delta \phi^{n}\right) \\
= & \left(f\left(\phi^{n+1}\right), \Delta \phi^{n+1}-\Delta \phi^{n}\right)+\left(f\left(\phi^{n}\right)-f\left(\phi^{n+1}\right), \Delta \phi^{n+1}-\Delta \phi^{n}\right)+\left(f^{\prime}\left(\phi^{n}\right)\left(\phi^{n+1}-\phi^{n}\right), \Delta \phi^{n}\right) \\
= & \left(f\left(\phi^{n+1}\right), \Delta \phi^{n+1}-\Delta \phi^{n}\right)+\left(-f^{\prime}(\eta)\left(\phi^{n+1}-\phi^{n}\right), \Delta \phi^{n+1}-\Delta \phi^{n}\right) \\
(3.28) & \quad+\left(f\left(\phi^{n+1}\right)-f\left(\phi^{n}\right)-\frac{f^{\prime \prime}(\xi)}{2}\left(\phi^{n+1}-\phi^{n}\right)^{2}, \Delta \phi^{n}\right) \\
& \quad\left(f\left(\phi^{n+1}\right), \Delta \phi^{n+1}-\Delta \phi^{n}\right)+\left(f\left(\phi^{n+1}\right)-f\left(\phi^{n}\right), \Delta \phi^{n}\right)+\left(-f^{\prime}(\eta)\left(\phi^{n+1}-\phi^{n}\right), \Delta \phi^{n+1}-\Delta \phi^{n}\right) \\
& \quad-\left(\frac{f^{\prime \prime}(\xi)}{2}\left(\phi^{n+1}-\phi^{n}\right)^{2}, \Delta \phi^{n}\right) .
\end{aligned}
$$

For term $I I I$, we derive

$$
\begin{aligned}
I I I: & =\left(f^{\prime}\left(\phi^{n}\right) f\left(\phi^{n}\right), \phi^{n+1}-\phi^{n}\right) \\
& =\left(f\left(\phi^{n}\right), f^{\prime}\left(\phi^{n}\right)\left(\phi^{n+1}-\phi^{n}\right)\right) \\
& =\left(f\left(\phi^{n}\right), f\left(\phi^{n+1}\right)-f\left(\phi^{n}\right)-\frac{f^{\prime \prime}(\xi)}{2}\left(\phi^{n+1}-\phi^{n}\right)^{2}\right) \\
& =\frac{1}{2}\left(\left\|f\left(\phi^{n+1}\right)\right\|^{2}-\left\|f\left(\phi^{n}\right)\right\|^{2}-\left\|f\left(\phi^{n+1}\right)-f\left(\phi^{n}\right)\right\|^{2}\right)-\left(f\left(\phi^{n}\right), \frac{f^{\prime \prime}(\xi)}{2}\left(\phi^{n+1}-\phi^{n}\right)^{2}\right) .
\end{aligned}
$$

For term $I V$, we derive

$$
\begin{aligned}
I V:=\left(A\left(\phi^{n}\right)-\alpha, \phi^{n+1}-\phi^{n}\right) & =\left(A\left(\phi^{n}\right)-\alpha\right)\left(\phi^{n+1}-\phi^{n}, 1\right) \\
& =\left(A\left(\phi^{n}\right)-\alpha\right)\left(A\left(\phi^{n+1}\right)-\alpha-\left(A\left(\phi^{n}\right)-\alpha\right)\right) \\
& =\frac{1}{2}\left(\left(A\left(\phi^{n+1}\right)-\alpha\right)^{2}-\left(A\left(\phi^{n}\right)-\alpha\right)^{2}\right)-\frac{1}{2}\left(A\left(\phi^{n+1}\right)-A\left(\phi^{n}\right)\right)^{2} .
\end{aligned}
$$


After combining the (3.24)-(3.26) and (3.28)-(3.30), we obtain

$$
\begin{aligned}
\frac{1}{2}\left\|\mathbf{u}^{n+1}\right\|^{2} & -\frac{1}{2}\left\|\mathbf{u}^{n}\right\|^{2}+\frac{1}{2}\left\|\mathbf{u}_{\star}^{n}-\mathbf{u}^{n}\right\|^{2}+\frac{1}{2}\left\|\tilde{\mathbf{u}}^{n+1}-\mathbf{u}_{\star}^{n}\right\|^{2} \\
& +\frac{1}{2} \delta t^{2}\left(\left\|\nabla p^{n+1}\right\|^{2}-\left\|\nabla p^{n}\right\|^{2}\right)+\nu \delta t\left\|\nabla \tilde{\mathbf{u}}^{n+1}\right\|^{2} \\
& +C_{1}^{n}\left\|\phi^{n+1}-\phi^{n}\right\|^{2}+C_{2}^{n}\left\|\nabla \phi^{n+1}-\nabla \phi^{n}\right\|^{2}+\delta t \frac{1}{M}\left\|\dot{\phi}^{n+1}\right\|^{2} \\
& +\frac{1}{2} \lambda \epsilon\left(\left\|\Delta \phi^{n+1}-f\left(\phi^{n+1}\right)\right\|^{2}-\left\|\Delta \phi^{n}-f\left(\phi^{n}\right)\right\|^{2}\right)+\frac{1}{2} \lambda \epsilon\left\|\Delta \phi^{n+1}-\Delta \phi^{n}\right\|^{2} \\
& +\frac{1}{2} \lambda M_{1}\left(A\left(\phi^{n+1}\right)-\alpha\right)^{2}-\left(A\left(\phi^{n}\right)-\alpha\right)^{2} \\
= & \lambda\left(-f^{\prime}(\eta)\left(\phi^{n+1}-\phi^{n}\right), \Delta \phi^{n+1}-\Delta \phi^{n}\right)\left(: A_{1}\right) \\
& -\lambda \epsilon\left(\frac{f^{\prime \prime}(\xi)}{2}\left(\phi^{n+1}-\phi^{n}\right)^{2}, \Delta \phi^{n}\right)\left(: B_{1}\right) \\
& +\frac{1}{2} \lambda \epsilon\left\|f\left(\phi^{n+1}\right)-f\left(\phi^{n}\right)\right\|^{2}\left(: C_{1}\right) \\
& +\lambda \epsilon\left(f\left(\phi^{n}\right), \frac{f^{\prime \prime}(\xi)}{2}\left(\phi^{n+1}-\phi^{n}\right)^{2}\right)\left(: D_{1}\right) \\
& +\frac{1}{2} \lambda M_{1}\left(A\left(\phi^{n+1}\right)-A\left(\phi^{n}\right)\right)^{2}\left(: E_{1}\right) .
\end{aligned}
$$

For terms on the right, we can derive

$$
\begin{aligned}
A_{1} & \leq L_{1} \lambda \epsilon\left\|\nabla \phi^{n+1}-\nabla \phi^{n}\right\|^{2}, \\
B_{1} & \leq \frac{1}{2} L_{2} \lambda \epsilon\left\|\Delta \phi^{n}\right\|_{\infty}\left\|\phi^{n+1}-\phi^{n}\right\|^{2}, \\
C_{1} & =\lambda \epsilon \frac{1}{2}\left\|f^{\prime}(\eta)\left(\phi^{n+1}-\phi^{n}\right)\right\|^{2} \leq \frac{1}{2} \lambda \epsilon L_{1}^{2}\left\|\phi^{n+1}-\phi^{n}\right\|^{2}, \\
D_{1} & \leq \frac{1}{2} \lambda \epsilon L_{2}\left\|f\left(\phi^{n}\right)\right\|_{\infty}\left\|\phi^{n+1}-\phi^{n}\right\|^{2}, \\
E_{1} & =\frac{1}{2} \lambda M_{1}\left(\int_{\Omega}\left(\phi^{n+1}-\phi^{n}\right) d x\right)^{2} \leq \frac{1}{2} \lambda M_{1} \mid \Omega\left\|\phi^{n+1}-\phi^{n}\right\|^{2} .
\end{aligned}
$$

Thus, if $C_{1}^{n}$ and $C_{2}^{n}$ satsify the condition (3.14), then the scheme has energy stability (3.15).

\section{Spatial DiscREtization}

In this paper, we take the finite element method for the spatial discretization to test the numerical scheme (3.8)-(3.12).

4.1. Weak formulation. It is noticed that bilaplacian operator in (3.8) will involve the second order derivative in the weak form when we take the trial function in $L^{2}$. To avoid the computational cost from the higher order finite element space, (3.8) was split into two second order elliptic equations [5].

First, we rewrite the equation (3.8) as follows.

$$
A_{2} \phi^{n+1}-B_{2} \Delta \phi^{n+1}+C_{2} \Delta^{2} \phi^{n+1}=G
$$


with the boundary conditions of $\left.\frac{\partial \phi}{\partial \mathbf{n}}\right|_{\partial \Omega}=0$ and $\left.\frac{\partial \Delta \phi}{\partial \mathbf{n}}\right|_{\partial \Omega}=0$, where $A_{2}=\frac{1}{M \delta t}+C_{1}^{n}+\frac{1}{\delta t(M+\delta t)\left|\nabla \phi^{n}\right|^{2}}$, $B_{2}=C_{2}^{n}, C_{2}=\lambda \epsilon$, and $G$ includes all explicit terms in (3.8) that reads as follows,

$$
\begin{aligned}
G= & \lambda \epsilon\left[\Delta f\left(\phi^{n}\right)+f^{\prime}\left(\phi^{n}\right) \Delta \phi^{n}-f^{\prime}\left(\phi^{n}\right) f\left(\phi^{n}\right)-\frac{M_{1}}{\epsilon}\left(A\left(\phi^{n}\right)-\alpha\right)+\frac{\phi^{n}-\delta t \mathbf{u}^{n} \cdot \nabla \phi^{n}}{\lambda \epsilon \delta t\left(M+\delta t\left|\nabla \phi^{n}\right|^{2}\right)}\right] \\
& +C_{1}^{n} \phi^{n}-C_{2}^{n} \Delta \phi^{n} .
\end{aligned}
$$

We introduce the parameter $a$ in the rewritten equation,

$$
-\left(a+\frac{B_{2}}{C_{2}}\right)\left(\Delta \phi^{n+1}-\frac{A_{2}}{a C_{2}+B_{2}} \phi^{n+1}\right)+\Delta\left(\Delta \phi^{n+1}+a \phi^{n+1}\right)=\frac{G}{C_{2}} .
$$

Let $-\frac{A_{2}}{a C_{2}+B_{2}}=a$. We get one value of $a$,

$$
a=\frac{-B_{2}-\sqrt{B_{2}^{2}-4 A_{2} C_{2}}}{2 C_{2}},
$$

with the requirement of $B_{2} \geq 2 \sqrt{A_{2} C_{2}}$. Let $\phi^{n+1}+a \phi^{n+1}=\psi^{n+1}$, thus, the fourth-order equation can be transformed into two decoupled Helmholtz-type equations,

$$
\begin{aligned}
& \Delta \psi^{n+1}-\left(a+\frac{B_{2}}{C_{2}}\right) \psi^{n+1}=\frac{G}{C_{2}}, \\
& \Delta \phi^{n+1}+a \phi^{n+1}=\psi^{n+1},
\end{aligned}
$$

and the boundary conditions can be transformed into

$$
\left.\frac{\partial \psi^{n+1}}{\partial \mathbf{n}}\right|_{\partial \Omega}=0,\left.\quad \frac{\partial \phi^{n+1}}{\partial \mathbf{n}}\right|_{\partial \Omega}=0 .
$$

The weak forms of (4.5) and (4.6) can be obtained by taking the inner product of both sides of these equations with a trial function $\varphi \in H^{1}$. Using integration by parts, the weak forms are given as follows. Find $\phi^{n+1} \in H^{1}(\Omega), \psi^{n+1} \in H^{1}(\Omega)$, such that for any $\varphi \in H^{1}(\Omega)$,

$$
\begin{aligned}
& -\left(\nabla \psi^{n+1}, \nabla \varphi\right)-\left(a+\frac{B_{2}}{C_{2}}\right)\left(\psi^{n+1}, \varphi\right)=\left(\frac{G}{C_{2}}, \varphi\right), \\
& -\left(\nabla \phi^{n+1}, \nabla \varphi\right)+a\left(\phi^{n+1}, \varphi\right)=\left(\psi^{n+1}, \varphi\right) .
\end{aligned}
$$

For the momentum equation (3.11), the corresponding weak formulation reads as follows. Find $\tilde{\mathbf{u}}^{n+1} \in H_{0}^{1}(\Omega)^{\operatorname{dim}}$, such that for any $\mathbf{v} \in H_{0}^{1}(\Omega)^{\operatorname{dim}}$,

$$
\left(\frac{1}{\delta t} \tilde{\mathbf{u}}^{n+1}+\left(\mathbf{u}^{n} \cdot \nabla\right) \tilde{\mathbf{u}}^{n+1}, \mathbf{v}\right)+\nu\left(\nabla \tilde{\mathbf{u}}^{n+1}, \nabla \mathbf{v}\right)=\left(\frac{1}{\delta t} \mathbf{u}_{\star}^{n}, \mathbf{v}\right)-\left(\nabla p^{n}, \mathbf{v}\right) .
$$

For the pressure equation (3.12), the corresponding weak formulation reads as follows. Find $p^{n+1} \in H_{c}^{1}(\Omega)=\left\{p: p \in H^{1}(\Omega), \int_{\Omega} p d x=0\right\}$, such that for any $q \in H_{c}^{1}(\Omega)$,

$$
\left(\nabla\left(p^{n+1}-p^{n}\right), \nabla q\right)=-\frac{1}{\delta t}\left(\nabla \cdot \tilde{\mathbf{u}}^{n}, q\right) .
$$

4.2. Finite element approximation. Let $S_{h} \subset H^{1}(\Omega)$ be the finite-dimensional subspace, which is constructed by the piecewise linear functions. 
Thus, $S_{h}^{0} \subset H_{0}^{1}(\Omega)$. Let $V_{\mathbf{u}_{h}}=\left(S_{h}^{0}\right)^{\operatorname{dim}}$ and $M_{h} \subset L_{0}^{2}(\Omega)$ be two finite-dimensional spaces satisfying the inf-sup condition,

$$
\inf _{q_{h} \in M_{h}} \sup _{\mathbf{u}_{h} \in V_{\mathbf{u}_{h}}} \frac{\int_{\Omega} q_{h} \nabla \cdot \mathbf{u}_{h} d x}{\left\|q_{h} \mid\right\| \mathbf{u}_{h} \|_{1}} \geq C,
$$

where $C>0$ independent of mesh size $h$ and $\left\|\mathbf{u}_{h}\right\|_{1}=\left\|\nabla \mathbf{u}_{h}\right\|+\left\|\mathbf{u}_{h}\right\|$.

Thus, we rewrite the equations (4.8)-(4.11) in finite element forms.

Step 1 , find $\phi_{h}^{n+1} \in S_{h}, \psi_{h}^{n+1} \in S_{h}$, such that for any $\varphi \in S_{h}$,

$$
\begin{aligned}
& -\left(\nabla \psi_{h}^{n+1}, \nabla \varphi\right)-\left(a+\frac{B_{2}}{C_{2}}\right)\left(\psi_{h}^{n+1}, \varphi\right)=\left(\frac{G_{h}}{C_{2}}, \varphi\right), \\
& -\left(\nabla \phi_{h}^{n+1}, \nabla \varphi\right)+a\left(\phi_{h}^{n+1}, \varphi\right)=\left(\psi_{h}^{n+1}, \varphi\right) .
\end{aligned}
$$

Step 2, Find $\tilde{\mathbf{u}}_{h}^{n+1} \in V_{\mathbf{u}_{h}}$, such that for any $\mathbf{v} \in V_{\mathbf{u}_{h}}$,

$$
\left(\frac{1}{\delta t} \tilde{\mathbf{u}}_{h}^{n+1}+\left(\mathbf{u}_{h}^{n} \cdot \nabla\right) \tilde{\mathbf{u}}_{h}^{n+1}, \mathbf{v}\right)+\nu\left(\nabla \tilde{\mathbf{u}}_{h}^{n+1}, \nabla \mathbf{v}\right)=\left(\frac{1}{\delta t} \mathbf{u}_{\star h}^{n}, \mathbf{v}\right)-\left(\nabla p_{h}^{n}, \mathbf{v}\right) .
$$

Step 3 , find $p_{h}^{n+1} \in M_{h}$, such that for any $q \in M_{h}$,

$$
\left(\nabla\left(p_{h}^{n+1}-p_{h}^{n}\right), \nabla q\right)=-\frac{1}{\delta t}\left(\nabla \cdot \tilde{\mathbf{u}}_{h}^{n+1}, q\right) .
$$

Then, we can obtain $\mathbf{u}_{h}^{n+1}$,

$$
\mathbf{u}_{h}^{n+1}=\tilde{\mathbf{u}}_{h}^{n+1}-\delta t \nabla\left(p_{h}^{n+1}-p_{h}^{n}\right)
$$

Remark 4.1. Here we show that we can easily prove the fully discrete scheme (4.13)-(4.17) satisfies the result of Theorem 3.1 when $\phi_{h}^{n+1} \in H^{2}(\Omega)$. However, it is difficult to prove the scheme is energy stable since integration by parts does not work as $\phi_{h}^{n+1} \in H^{1}(\Omega)$ here.

4.3. Preconditioning. Using the linear element in the finite element method, the first and third terms in equation (4.15) lead to sparse and symmetric matrices, but the second term in equation (4.15) leads to asymmetric matrices. Here we do not explicitly build it, instead use the Preconditioned Conjugate Gradient (PCG) method. The matrices for left hand side of the following equation will be used as preconditioner of (4.15).

Find $\tilde{\mathbf{u}}_{h}^{n+1} \in V_{\mathbf{u}_{h}}$, such that for any $\mathbf{v} \in V_{\mathbf{u}_{h}}$,

$$
\frac{1}{\delta t}\left(\tilde{\mathbf{u}}_{h}^{n+1}, \mathbf{v}\right)+\nu\left(\nabla \tilde{\mathbf{u}}_{h}^{n+1}, \nabla \mathbf{v}\right)=r_{1}[\mathbf{v}],
$$

where the vector $r_{1}[\mathbf{v}]$ is the residual of (4.15).

\section{Numerical Simulations}

In this section, we present some numerical experiments using the schemes constructed in section 3 and 4. We use the inf-sup stable $I s o-P 2 / P 1$ element [33] for the velocity and pressure, and linear element for the phase function $\phi$. 
5.1. Accuracy test. Example 1. We first test the convergence rates of the proposed schemes (3.8)-(3.12). In $\Omega=[0,2]^{2}$, we set the exact solution as

$$
\left\{\begin{array}{l}
\phi(t, x, y)=2+\cos (\pi x) \cos (\pi y) \sin t \\
u(t, x, y)=\pi \sin (2 \pi y) \sin ^{2}(\pi x) \sin t \\
v(t, x, y)=-\pi \sin (2 \pi x) \sin ^{2}(\pi y) \sin t \\
p(t, x, y)=\cos (\pi x) \sin (\pi y) \sin t
\end{array}\right.
$$

We choose $\epsilon=0.025, \nu=1, M=1, \lambda=10^{-7}, M_{1}=0$. Some suitable force fields are imposed such that the given solutions satisfy the coupled systems. In order to test the accuracy for time, we used 10145 nodes and 19968 triangle elements such that the spatial error are sufficient small. In Figure 5.1, we plot the $L^{2}$ errors of the velocity, pressure and phase function between the numerical solution and the exact solution at $t=1$ with different time step size $\delta t=0.0001,0.0005,0.001$, 0.005 , and 0.01 . The numerical results show that scheme (3.8)-(3.12) is first-order accurate in time for all variables.

5.2. The deformations of a red blood cell. In all next examples, we simulate the deformation of a single red blood cell (RBC) when it travels in a vessel as a capillary tube with a narrow portion by considering the membrane of the RBC as a vesicle with elasticity. The background fluid is assumed to be Newtonian fluids.

To simulate the blood vessel, the physical domain $\Omega$ is considered to be a cylinder, where a semi-circular hole is eliminated on the side of the cylinder domain representing the narrow portion of the vessel. We also assume that all variables are axisymmetric. For the implementation of the cylindrical coordinates $(r, \theta, z)$, the Navier-Stokes equations in the system read as in $[19,26]$. Without considering the azimuthal $\theta$ direction, the system can be considered to be two-dimensional problems briefly.

The domain $\Omega$ is cut into 36608 triangle elements by MATLAB. The main parameters are set as follows,

$$
\nu=0.01, M=1, \epsilon=0.0125, \delta t=0.001, M_{1}=10^{7}, M_{2}=1000 .
$$

The initial values of the flow fields along the $\mathrm{r}$ and $\mathrm{z}$ directions are $\mathbf{u}_{0}=\left(u_{0}, w_{0}\right)$ where $u_{0}=0$ and $w_{0}=100$ at $r=0, w_{0}=0$ otherwise.

Example 2: The deformation of a cell with smaller surface tension. We set $\lambda=10^{-7}$ in this example. In Figure. 5.2, when the cell goes toward the narrow portion, it starts to deform at the time $T=0.5$. Soon the cell flattens horizontally from $T=1$ to $T=2$. When it totally passes over the narrow portion, the cell recovers to spherical shape after $T=3$.

The discrete energy of the system is plotted in Figure 5.3. We observe that the discrete energy of the system is dissipated with respect to the time.

Example 3: The deformation of a cell with bigger surface tension. We take $\lambda=3 \times 10^{-7}$ that is slightly bigger than Example 2. In Figure 5.4, the cell travels relatively slower than Example 2. Due to the affections of the surface tension, for the same time period up to $T=4$, the cell cannot totally pass through the narrow part. The discrete energy of the system is plotted in Figure 5.5, that shows the discrete energy is dissipated with the time.

For different surface tension parameters, we plot the energy dissipation curve in Figure 5.6.

Example 4: The deformation of a biconcave shape cell in longer vessel. We choose the biconcave configuration to be the initial shape of red blood cell in a longer cylindrical domain as in [21]. A semi-circular hole is still eliminated on the side of the cylinder to represent the narrow portion of the vessel. The dynamics of the deformations are shown in Figure 5.7. At $T=0.2$, 
the deformation starts when the cell flows toward the narrow portion. When the cell tries to pass through the narrow part of the capillary from $T=0.8$ to $T=0.1$, due to the elasticity of the membrane, the cell is elongated to largely deformed crescent-like shape, and then passes over. This kind of capability of deformations are essential features of the blood flow mechanics. Our numerical results are qualitatively consistent with the numerical simulation in [21], where, a different model (spring network membrane model) was used.

In Figure 5.8, we cite the biological experiments, where a red blood cell will become a crescent-like shape when it travels from the wide part of a capillary to the narrow part. This is also qualitatively consistent with our numerical simulations.

\section{Conclusions AND REMARKS}

In this paper we construct a stabilized, decoupled, time discretization numerical scheme for the phase-field vesicle membrane model. Even though the model was proposed over a decade and applied in many literatures $[6-8,10]$, however, there are still not any energy stable schemes available.

Our scheme is unconditionally energy stable, and is quite easy to implement. At each time step, one only needs to solve Poisson type equations due to the decoupling and linearity. All nonlinear terms are simply treated explicitly. The fourth order bilaplacian operator is split into two second order equations. We also present ample numerical simulations to illustrate the efficiency of the proposed scheme, and to match to the experimental benchmark.

\section{ACKNOWLEDGMENTS}

G. Ji is partially supported by NSFC-11271048 and Fundamental Research Funds for the Center University. H. Zhang is partially supported by NSFC-RGC-11261160486, NSFC-11471046 and the Ministry of Education Program for New Century Excellent Talents Project NCET-12-0053. X. Yang is partially supported by NSF DMS-1418898, NSF DMS-1200487, AFOSR FA9550-12-1-0178, SC Epscor Gear fund and NSFC-11471312.

\section{REFERENCES}

[1] F. Boyer AND S. Minjeaud, Numerical schemes for a three component Cahn-Hilliard model, ESAIM Math. Model. Numer. Anal., 45 (2011), 697-738.

[2] J. W. Cahn And S. M. Allen, A microscopic theorey for domain wall motion and its experimental verification in fe-al alloy domain growth kinetics, J. Phys. Colloque, C7 (1977), pp. C7-51.

[3] P.G. Ciarlet, Introduction to Linear Shell Theory, Series in Applied Mathematics (Paris), vol. 1, GauthierVillars, Editions Scientifiques et Medicales Elsevier, Paris, (1998).

[4] P.G. Ciarlet, Mathematical elasticity, vol. III, in: Studies in Mathematics and its Applications, vol. 29, NorthHolland, Amsterdam, (2000), Theory of shells.

[5] S. Dong And J. Shen, A time-stepping scheme involving constant coefficient matrices for phase-field simulations of two-phase imcompressible flows with large density ratios, J. Comput. Phys., 231 (2012), 5788-5804.

[6] Q. Du, C. LIU AND X. WANG, A phase field approach in the numerical study of the elastic bending energy for vesicle membranes, J. comput. Phys., 198 (2004), 450-468.

[7] Q. Du, C. LiU AND X. WANG, Retriving topological information for phase field models, SIAM J. Appl. Math., 65 (2005), 1913-1932.

[8] Q. Du, C. Liu, R. Ryham And X. Wang, Modeling the spontanous curvature effects in static cell membrane deformations by a phase field formulation, Commun. Pure Appl. Analy., 4 (2005), 537-548.

[9] P.C. Hohenberg And B.I. Halperin, Theory of dynamic critical phenomena, Rev. Mod. Phys. 49(1977), 435479.

[10] Q. Du, C. Liu, R. Ryham And X. Wang, A phase field formulation of the Willmore problem, Nonlinearity, 18 (2005), 1249-1267. 
[11] J. Hua, P. Lin, C. Liu, And Q. WAng, Energy law preserving c0 finite element schemes for phase field models in two-phase flow computations, J. of Comput. Phys., 230 (2011), 7115-7131.

[12] H. Johnston And J. G. Liu, Accurate,stable and efficient Navier-Stokes solvers based on explicit treatment of the pressure term, J. Comput. Phys., 199 (2004), 221-259.

[13] Daniel Kessler, R. H. Nochetto And A. Schmidt, A posteriori error control for the Allen-Cahn problem : circumventing Gronwall's inequality, M2AN Math. Model. Anal., 38 (2004), 129-142.

[14] R. Lipowsky, The morphology of lipid menbranes current opinion in structural biology, Comm. Pure Appl. Math., XLVIII (1995), 501-537.

[15] C. Liu And J. Shen, A phase field model for the mixture of two incompressible fluids and its approximation by a fourier-spectral method, Physica D, 179 (2003), 211-228.

[16] C. Liu, J. Shen AND X. YANG, Dynamics of defect motion in nematic liquid crystal flow: modeling and numerical simulation, Commun. Comput. Phys., 2 (2007), 1184-1198.

[17] C. Liu, J. Shen And X. YAng, Decouppled Energy stable schemes for a phase-field model of two-phase incompressible flows with variable density, J. Sci. Comput., 62 (2015), 601-622.

[18] J. Li AND Y. RENARDY, Numerical study of flows of two immiscible liquids at low reynolds number, SIAM Reviw, $42(2000), 417-439$.

[19] J. M. Lopez, F. Maques And J. Shen, An efficent spectral-projection method for the Navier-Stokes equations in cylidrical geometries, J. Comput. Phys., 139 (1998), 308-326.

[20] S. Minjeaud, An unconditionaly stable uncoupled shceme for a triphasic Cahn-Hilliard/Navier-Stokes model, Commun. Comput. Phys., 29 (2013), 584-618.

[21] P. G. H. Nayanajith1, S. C. Saha, And Y. T. Gu, Deformation properties of single red blood cell in a stenosed microchannel, APCOM ISCM 11-14th December, (2013), Singapore.

[22] J. Pyo And J. Shen, Gauge-uzawa methods for incompressible flows with variable density, J. Comput. Phys., 221 (2007), 181-197.

[23] Z.H. QiaO, Z. Zhang AND T. TANG, An adaptive time-stepping strategy for the molecular beam epitaxy models, SIAM J. Sci. Comput., 33 (2011), 1398-1414.

[24] Z.H. QIAO, S.Y. Sun, Two-phase fluid simulation using a diffuse interface model with Peng-Robinson equationof a state, SIAM J. Sci. Comput., 36 (2014), 708-728.

[25] Z.H. QIAO, T. TANG AND H.H. XIE, Error analysis of a mixed finite element method for the molecular beam epitaxy model, SIAM J. Numer. Anal., 53 (2015), 184-205.

[26] J. SHEn, Efficient spectral-galerkin method III:polar and cylindrical geometries, SIAM J. Sci. Comput., 18 (1997), $1583-1604$.

[27] J. SHEN, Modeling and numerical approximaton of two-phase incompressible flows by a phase-field approach, In Multiscal Modeling and Anaysis for Materials Simulation, Lecture Note Serires, Vol. 9. IMS, National University of Singapore, (2011), Edited by W. Bao and Q. Du, 147-196.

[28] J. ShEn AND X. YANG, An efficient moving mesh spectral method for the phase-field model of two-phase flows, J. Comput. Phys., 288 (2009), 2978-2992.

[29] J. Shen AND X. YAng, Energy stable schemes for Cahn-Hilliard phase-field model of two phase incompressible flows, Chin. Ann. Math., Series B, 31 (2010), 743-758.

[30] J. Shen And X. Yang, Numerical approximations of Allen-Cahn and Cahn-Hilliard equations, Disc. Conti. Dyn. Sys., Series A, 28 (2010), 1669-1691.

[31] J. Shen AND X. YAng, Decoupled energy stable schemes for phase-field models of two-phase complex fluids, SIAM J. Sci. Comput., 36 (2014), 122-145.

[32] J. Shen AND X. YAng, Decoupled, energy stable schemes for phase-field models of two-phase incompressible flows, SIAM J. Numer. Anal., 53 (2015), 279-296.

[33] M. Tabata And D. Tagamai, Error estimates for finite element approximations of drag and lift in nonstantionary Navier-stokes flows, Jpn. J. Indust. Appl. Math., 17 (2000), 371-389.

[34] J. D. VAN DER WAALS, The thermodynamic theory of capillarity under the hypothesis of a continuous variation of density, Verhandel Konink, Akad. Weten. Amsterdam,(Sect.1)1(no. 8), 1C56 (in Dutch), 1892. Translation by J. S. Rowlingson,J. Statist. Phys. 20(1979), 197-244.

[35] C.J. Xu AND T. TANG, Stability analysis of large time-stepping methods for epitaxial growth models, SIAM J. Numer. Anal., 44 (2006), 1759-1779. 
[36] P. Yue, J. J. Feng, C. Liu, and J. Shen, A diffuse-interface method for simulating two-phase flows of complex fluids, J. Fluid Mech., 515 (2004), 293-317.

[37] Z. Zhang, Y. Ma AND Z. QIAO, An adaptive time-stepping strategy for solving the phase field crystal, J. Comput. Phys., 249 (2013), 204-215. 


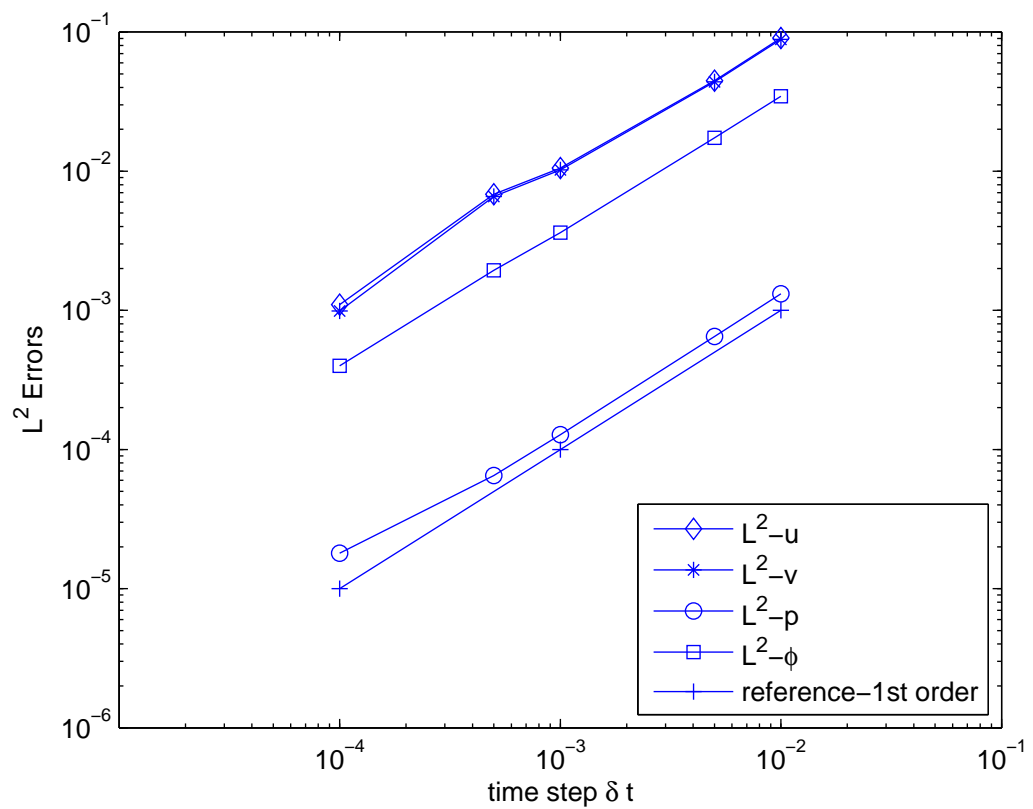

Figure 5.1. Example 1. Temporal convergence rates of $L^{2}$ errors for the velocity $(\mathbf{u}=(u, v))$, pressure $p$, and phase field function $\phi$ as the function of time step $\delta t$. 


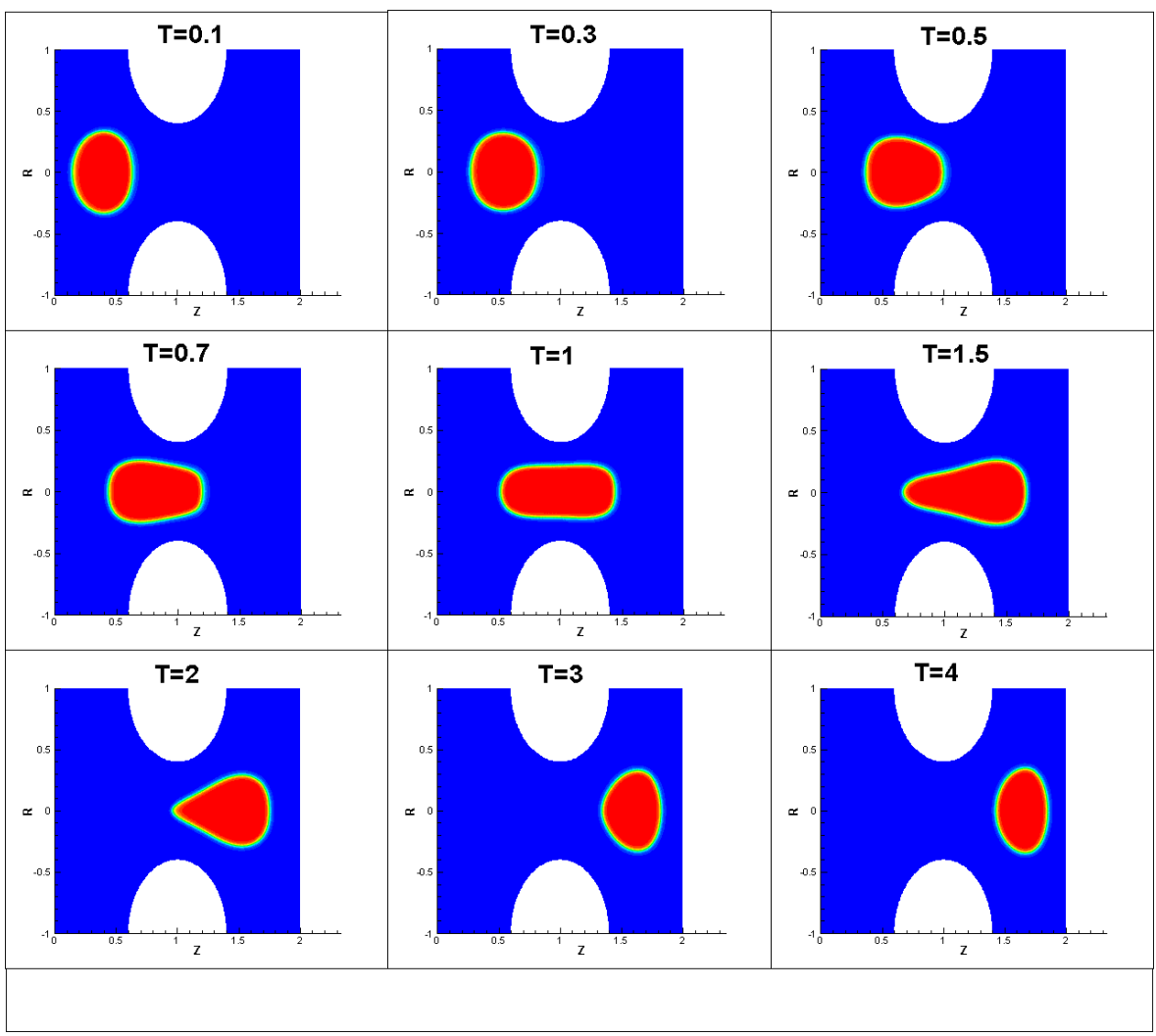

Figure 5.2. Example 2. The snapshots of the deformation dynamics of the cell with $\lambda=10^{-7}$.

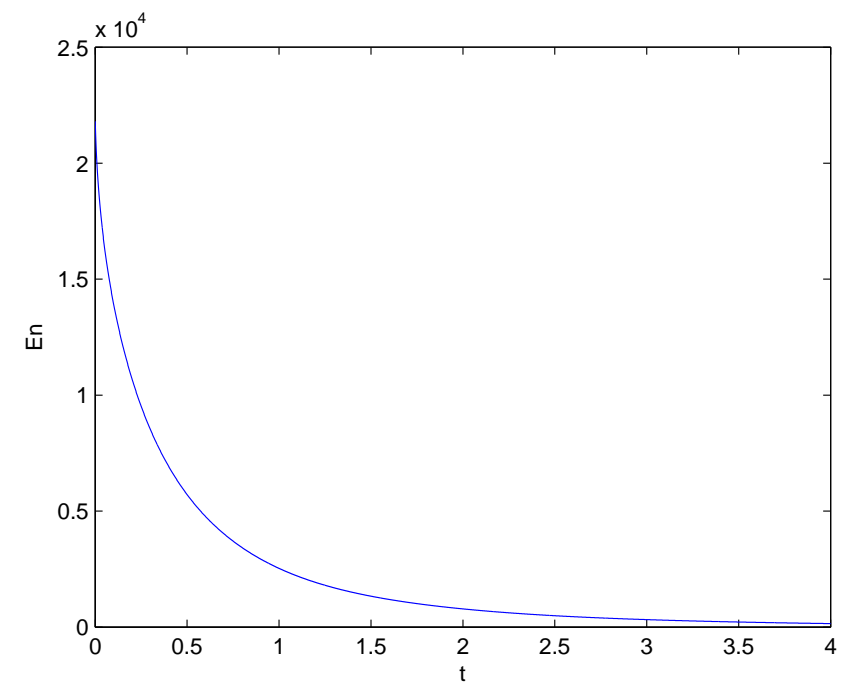

Figure 5.3. Example 2. The time evolution of the energy. 


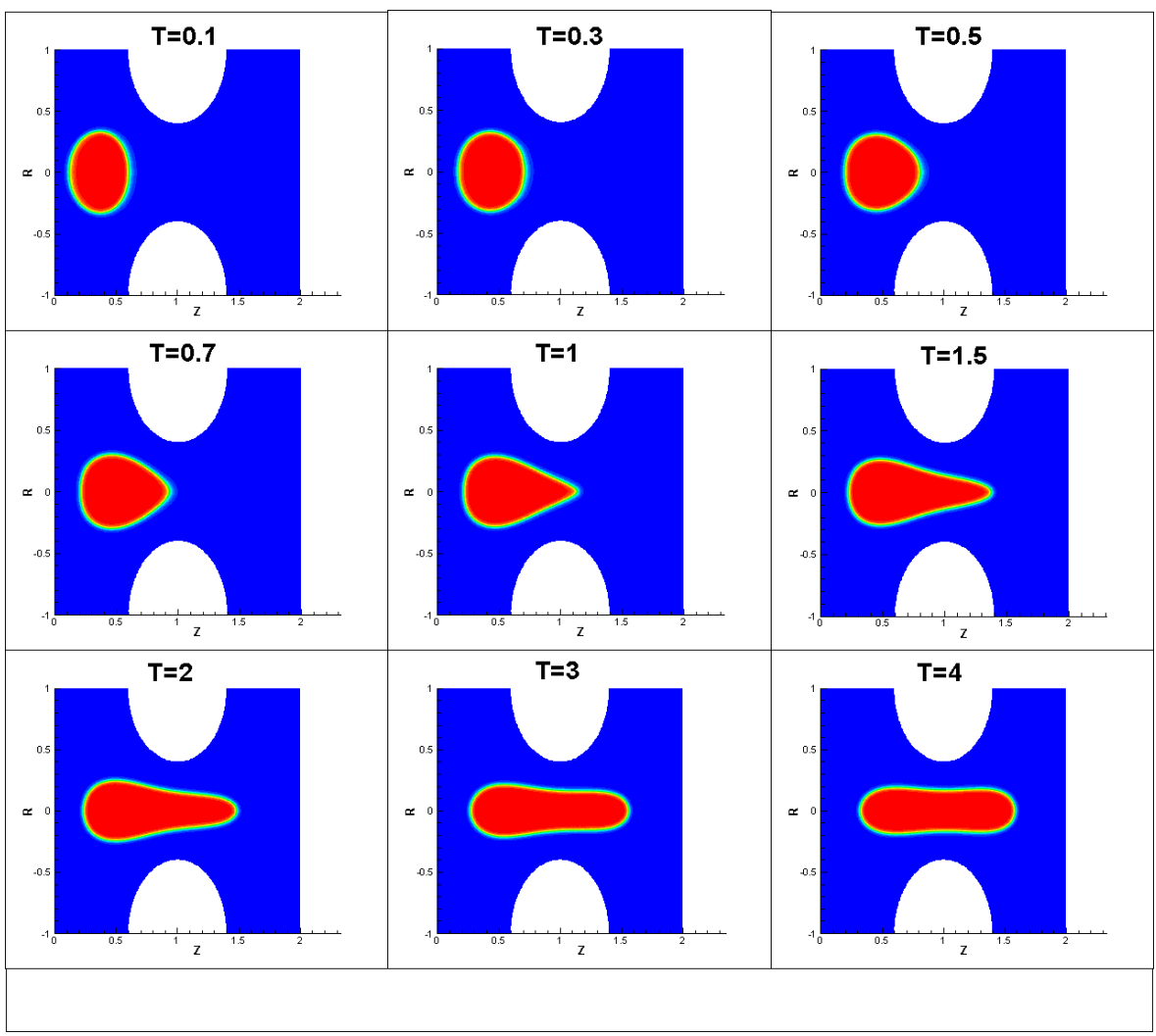

Figure 5.4. Example 3. The snapshots of the deformation dynamics of the cell with $\lambda=3 \times 10^{-7}$.

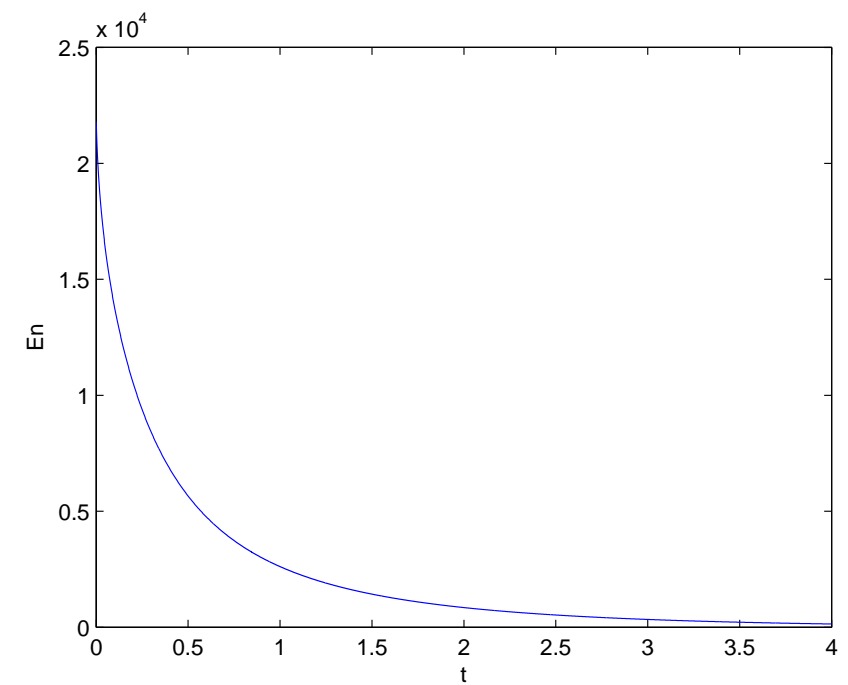

Figure 5.5. Example 3. The time evolution of the energy. 

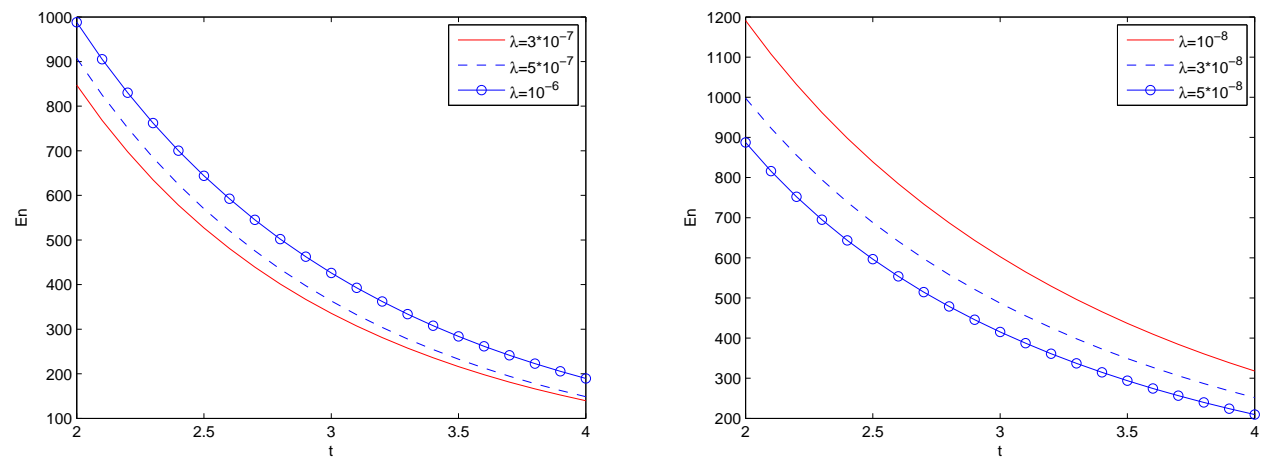

Figure 5.6. The time evolution of the energy of the system with different surface tension parameters $\lambda$.

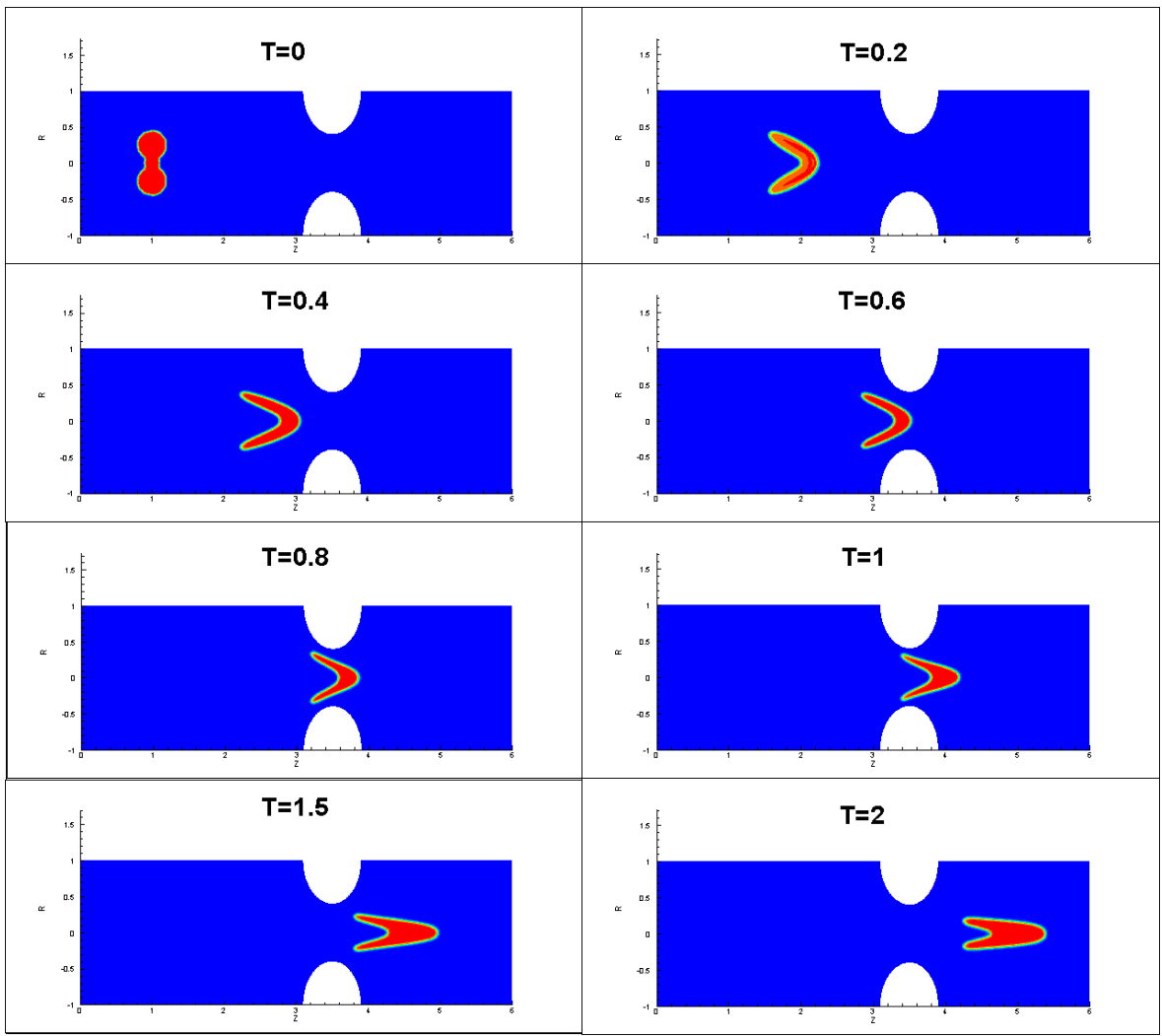

Figure 5.7. Example 4. The deformation of a cell when it passes through the narrow portion of the vessel at $T=0,0.2,0.4,0.6,0.8,1,1.5,2$. 


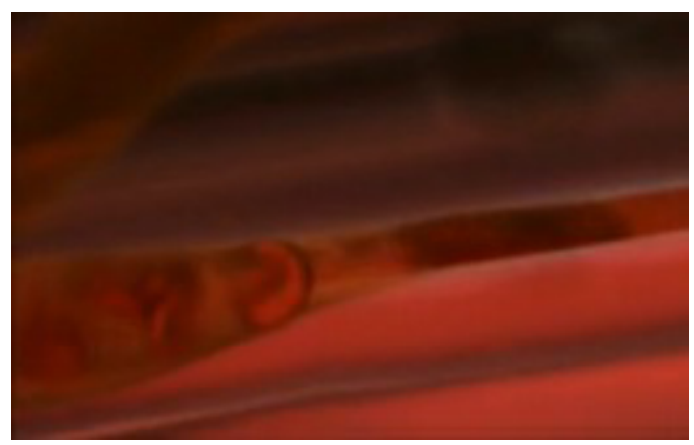

FiguRE 5.8. Experimental photo of a cresent-shape red blood cell when it travel through the narrow vessel. (http://www.thevisualmd.com/center.php?idg=5216) 